\title{
aB-crystallin inhibits amyloidogenesis by disassembling aggregation nuclei
}

\author{
Olga Tkachenko ${ }^{1}$, Justin L.P. Benesch ${ }^{1 \dagger}$, Andrew J. Baldwin ${ }^{1 \dagger}$

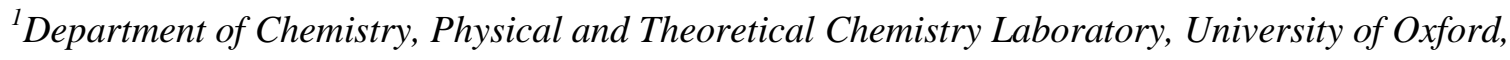 \\ South Parks Road, Oxford, OX1 3QZ, U.K. \\ ${ }^{\dagger}$ Correspondence to: justin.benesch@chem.ox.ac.uk and andrew.baldwin@chem.ox.ac.uk
}

Keywords: NMR spectroscopy, molecular chaperones, small heat-shock proteins, $\alpha B$-crystallin, HSPB5, $\alpha$-lactalbumin, amyloid inhibition mechanism 


\begin{abstract}
Amyloid formation is implicated in a range of neurodegenerative conditions including Alzheimer's and Parkinson's diseases. The small heat-shock protein $\alpha \mathrm{B}$-crystallin $(\alpha \mathrm{BC})$ is associated with both, and directly inhibits amyloid formation in vitro and its toxicity in cells. Studying the mechanism of aggregation inhibition is challenging owing to sample heterogeneity and the dynamic nature of the process. Here, by means of NMR spectroscopy and chemical kinetics, we establish the mechanism by which the protein $\alpha$-lactalbumin aggregates and forms amyloid, and how this is inhibited by $\alpha \mathrm{BC}$. In particular, we characterise the lifetime of the unstable aggregation nucleus, and determine that this species is specifically destabilised by $\alpha \mathrm{BC}$. This mechanism allows the chaperone to delay the onset of aggregation, although it is overwhelmed on longer timescales. The methodology we present provides a mechanistic understanding of how $\alpha \mathrm{BC}$ reduces the toxicity of amyloids, and is widely applicable to other complex mixtures.
\end{abstract}
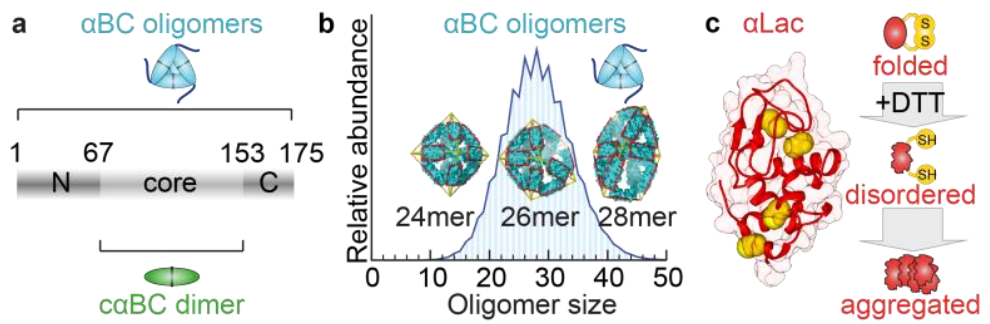

Figure 1 The chaperone $\alpha \mathrm{BC}$ and the aggregating client $\alpha$ Lac. a The core domain of $\alpha \mathrm{BC}$ is flanked by disordered $\mathrm{N}$ - and $\mathrm{C}$ - termini. The excised core exists as a dimer $(\mathrm{c} \alpha \mathrm{BC})$. Both full-length $\alpha \mathrm{BC}$ and $\mathrm{c} \alpha \mathrm{BC}$ are potent chaperones ${ }^{1}$. b Full-length $\alpha \mathrm{BC}$ exists as a polydisperse ensemble and structural models are available for the principally populated oligomers $^{2}$. c Structure of apo $\alpha$ Lac with the four disulphide bonds highlighted in yellow. Addition of DTT leads to reduction of the disulphides and adoption of a disordered conformation, which, under the conditions tested here, aggregates to form amyloid. 


\section{Introduction}

Neurodegenerative conditions such as Parkinson's and Alzheimer's diseases (PD and AD) are some of the greatest health challenges for the ageing world population ${ }^{3}$. These conditions are associated with aggregation of protein in the form of amyloid, which can be highly toxic ${ }^{4,5}$. Cells possess a sophisticated defence network against such protein aggregates that includes the small heat-shock proteins (sHSP) ${ }^{6-9}$. In humans, the sHSP $\alpha \mathrm{B}$-crystallin $(\alpha \mathrm{BC})$ is linked to $\mathrm{AD}$ and $\mathrm{PD}^{10}$, being upregulated in neurons and glia adjacent to amyloids in humans ${ }^{11-13}$, and found within plaques analysed ex vivo ${ }^{12,14}$. In vitro, $\alpha \mathrm{BC}$ significantly inhibits amyloid formation of a wide range of proteins ${ }^{10}$. The molecular mechanism of this process, comprising a description of the kinetic and thermodynamic relationships between interacting species, has proven highly challenging to obtain owing to the heterogeneity and kinetic instability of complexes formed between protein aggregates and chaperones.

Amyloid formation typically begins from monomeric protein, culminating in high order aggregates. In principle, chaperones could associate with any, or all, of these species along the amyloidogenesis pathway. A wide range of biophysical techniques including light scattering, changes in fluorescence of histological dyes, circular dichroism, size exclusion chromatography, ultracentrifugation, native mass spectrometry, small-angle X-ray scattering, and nuclear magnetic resonance (NMR) spectroscopy have been employed to determine features of this process ${ }^{5,10,15-25}$. Significant insights into how proteins aggregate, and how chaperones attenuate aggregation, have been obtained by relating such data to analytical equations in specific regimes ${ }^{26-28} \cdot \alpha \mathrm{BC}$ is a particularly challenging chaperone to study, as it spontaneously assembles into a polydisperse ensemble of exchanging oligomers $^{29-31}$ (Fig. 1). If the N- and C-terminal regions are removed, the excised core domain (caBC) forms dimers that inhibit amyloid formation similarly to the full-length, oligomeric form of the protein $^{1}$. Despite extensive research efforts, the mechanism by which these forms of $\alpha \mathrm{BC}$ interact with substrate to inhibit amyloid formation has remained elusive.

Here, we present a novel strategy for mechanism determination based on combining NMR spectroscopy with numerical chemical kinetic modelling and stepwise regression. With differential isotope labelling, our NMR experiments allow us to monitor changes in both the chaperone and the 
amyloid-forming protein directly and independently. By analysing datasets acquired over a range of concentrations, we can test a variety of possible numerical aggregation models, to find those that best fit the data. Through quantitative study of the effects of $\alpha \mathrm{BC}$ on a model amyloid-forming protein, bovine $\alpha$-lactalbumin $\left(\alpha\right.$ Lac, Fig. 1c) ${ }^{16}$, with our method, we determine a mechanism for how a model amyloid-forming system aggregates, and how this is inhibited by $\alpha B C$.

Our analysis reveals that $\alpha$ Lac aggregates via two parallel nucleated pathways, operating on different timescales. We find that, in addition to binding mature aggregates, as has been observed previously $^{20,21,32}$, the action of $\alpha \mathrm{BC}$ is dominated by disrupting lowly populated aggregation nuclei, thus redirecting the substrate towards the monomeric form and thereby slowing aggregation. While both $\alpha \mathrm{BC}$ and $\mathrm{c} \alpha \mathrm{BC}$ display a similar mechanism, we find that the full-length protein interacts only with aggregated forms of $\alpha \mathrm{Lac}$, while $\mathrm{c} \alpha \mathrm{BC}$ additionally interacts with free monomers. This interaction with unaggregated monomers would be undesirable in the context of a cell and provides a rationale for why $\alpha \mathrm{BC}$ forms oligomers. Remarkably, while both forms of $\alpha \mathrm{BC}$ attenuate aggregation in a concentration-dependent manner, we find that at longer times both proteins are effectively overwhelmed, and that there a limited window of time and client concentration in which $\alpha \mathrm{BC}$ is effective. This provides quantitative insight into how $\alpha \mathrm{BC}$, and potentially other sHSPs, may be involved in neurodegenerative disorders, including AD and PD. Our combined NMR and chemical kinetics methodology is applicable to the mechanistic study of other complex and heterogeneous mixtures, and provides a quantitative foundation for therapy design. 


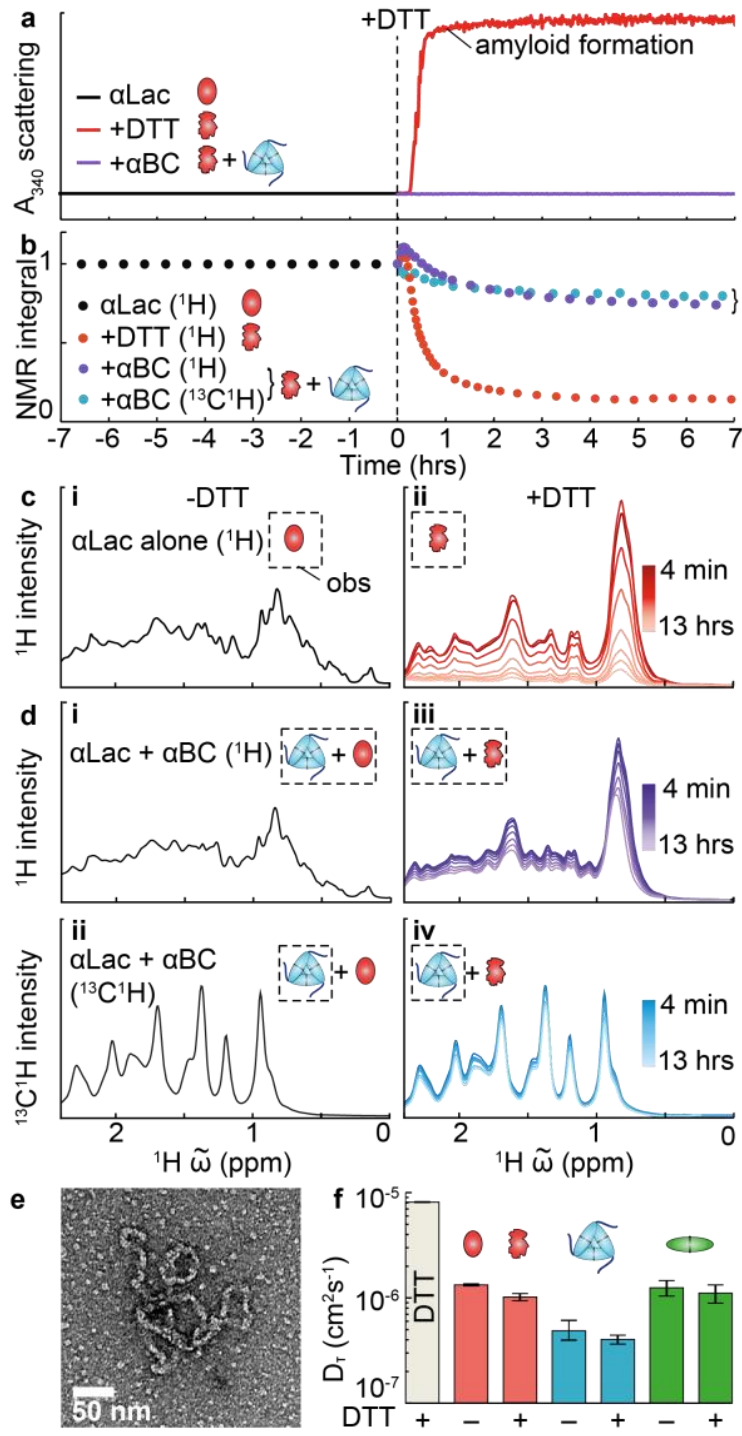

Figure 2 | Following amyloid formation of $\alpha \mathrm{Lac}$ by light scattering, and NMR. a Aggregation of $200 \mu \mathrm{M} \alpha \mathrm{Lac}$ followed by light scattering in the absence (red) and presence (purple) of $\alpha \mathrm{BC}$ at a $1: 1$ molar ratio. b The same process followed by NMR, where the ${ }^{13} \mathrm{C}$ isotopically enriched chaperone can be monitored independently. $\mathbf{c} \mathbf{i}^{1} \mathrm{H}$ NMR spectra of $\alpha \mathrm{Lac}$ prior to addition of DTT in the absence of chaperones. The observed species (obs) is illustrated in a dashed box. ii Time evolution of the $\alpha \mathrm{Lac}{ }^{1} \mathrm{H}$ spectra following addition of DTT. $\mathbf{d}^{1} \mathrm{H}$ (i) and ${ }^{13} \mathrm{C}$-edited ${ }^{1} \mathrm{H}$ spectra (ii) of the $\alpha \mathrm{Lac} / \alpha \mathrm{BC}$ mixture prior to the addition of DTT. Both species are observed in $\mathbf{i}$, whereas only chaperone is observed in ii. The evolution of both spectra following DTT addition is shown in iii $\left({ }^{1} \mathrm{H}\right)$ and iv $\left({ }^{13} \mathrm{C}\right.$-edited $\left.{ }^{1} \mathrm{H}\right)$. The time-course of the integrated signal from these spectra is shown in b. e Negative stain electron microscopy showing aggregates of $\alpha \mathrm{Lac}$ in the absence of $\alpha \mathrm{BC}$ following 12 hours of exposure to DTT. f Translational diffusion coefficients of the species observed measured by NMR. The values obtained are consistent with the spectra being dominated by the free, unbound species being observed at all times (see also Fig. S3). 


\section{Results}

\section{A quantitative NMR assay for monitoring protein aggregation}

To obtain a reference data set, we performed a static light scattering assay, a widely used in vitro measure of sHSP chaperone activity ${ }^{10}$. Consistent with previous data ${ }^{1,16,33}$, upon addition of the reductant dithiothreitol (DTT) to $\alpha$ Lac, a sigmoidal increase in scattering was observed (Fig. 2a). The resulting aggregates were found to be amyloid (Fig. S1). Flexible fibrillar aggregates with $\beta$-sheet secondary structure were observed in electron microscopy (EM) images (Fig. 2e) that bound the histological dye thioflavin T (ThT) (Fig. S1). Addition of an equimolar amount of either full-length oligomeric $\alpha \mathrm{BC}$ or $\mathrm{c} \alpha \mathrm{BC}$ resulted in complete abrogation of the increase in scattering over the timescale of the experiment (Fig. 2a). These results clearly demonstrate the potency of the chaperone in inhibiting amyloid formation.

Light scattering has a complex dependence on the shape, size, and distribution of the aggregating particles, making it challenging to analyse such data quantitatively to obtain a molecular mechanism. By contrast, NMR spectroscopy is amenable to quantitative studies of amyloid formation, as the observed signal can be directly related to the concentration of observed particles ${ }^{22,34}$. In the absence of DTT, the ${ }^{1} \mathrm{H}$ NMR spectrum of $\alpha \mathrm{Lac}(200 \mu \mathrm{M})$ is typical of a folded, globular protein ${ }^{35}$ (Fig. 2c, i). Upon the addition of DTT, the spectrum changes in appearance and returns a $\sim 20 \%$ decrease in the translational diffusion coefficient (Fig. 2c ii; f). At this $\alpha$ Lac concentration, the conformational change results in an increase in overall signal intensity to a maximum within minutes, within the apparent lag phase of the light scattering measurements. These observations are consistent with the formation of a disordered "molten globule" ${ }^{36-39}$. The signal then decays on a timescale of hours to a steady-state value. This is consistent with expectations that high molecular mass aggregates are formed over time that are not directly observable by solution NMR owing to their relatively rapid transverse relaxation $^{16,40}$. We found the data to be very reproducible at a range of $\alpha$ Lac concentrations between 100-2000 $\mu \mathrm{M}$ (Fig. S2). At higher concentrations, the initial rise in signal intensity was attenuated, the signal decay rate increased, and the steady-state relative abundance of monomer 
decreased (Fig. S2). The total $\alpha$ Lac concentration dependence of residual monomer levels at the end of the experiment demonstrates that $\alpha$ Lac aggregation must be a reversible process, or else the signal ought to decay to zero as all monomers are converted into aggregates ${ }^{23}$. These results demonstrate that the NMR experiment provides a simple assay to follow the change in free monomer concentration quantitatively and in real time.

\section{Selective isotope labelling allows independent monitoring of chaperone activity}

To extend our approach to a multi-component mixture, we employed a selective isotope-labelling strategy so that, by performing isotope-edited NMR experiments, we would be able to monitor the components independently. For full-length $\alpha \mathrm{BC}$, we introduced ${ }^{13} \mathrm{C}$ labels, allowing us to specifically follow the flexible C-terminal region on the exterior of the oligomers ${ }^{41-43}$. For $c \alpha \mathrm{BC}$, we employed ${ }^{15} \mathrm{~N}$ enrichment, allowing us to follow each position in the backbone of the protein. These were mixed individually with unlabelled $\alpha$ Lac. We could therefore monitor both the chaperone and amyloid formation independently, without the need for extrinsic labels (Fig. 2b and d, Fig. S2). Prior to addition of DTT, the ${ }^{1} \mathrm{H}$ spectra of the mixtures were the sum of the spectra of the individual components (Fig. S3). After the addition of DTT, even at the earliest times, where the light scattering traces in the presence or absence of chaperone are indistinguishable (and approximately zero, Fig. 2a), significant changes were seen in the NMR spectra, revealing that the experiment is sensitive to early events in aggregation (Fig. S2). In addition, the rate of $\alpha$ Lac signal decay was substantially slower in the presence of chaperone, indicative of chaperone activity (Fig. 2b).

No significant change was observed in either the position or linewidth of resonances in twodimensional isotope-edited NMR spectra of either chaperone (Fig. S3). Instead, a uniform loss of signal intensity was observed across the spectrum (Fig. 2d, Fig. S3) indicating that the observed species are in the 'slow' regime of chemical exchange ${ }^{34}$. Similarly to $\alpha$ Lac alone, diffusion experiments revealed that the observed species were free in solution, rather than trapped in aggregates (Fig. 2f and S3), and that the loss in signal intensity coincided with the build-up of high molecularmass aggregates visible in EM images (Fig. S1). 
We acquired a large dataset of interleaved NMR experiments by varying $\alpha$ Lac concentration systematically (Fig. S2). Both ${ }^{1} \mathrm{H}$ and isotope-edited NMR signal intensities reached non-zero, concentration-dependent steady-state values at longer times, revealing the reversible nature of chaperone- $\alpha$ Lac interactions (Fig. S2). Significantly more signal was observed at longer times after incubation with chaperone, indicating enhanced protection (Fig. S2). The visible differences in the data effected by the addition, and variation, of chaperone reveal that the NMR experiment is information-rich, and sensitive to molecular processes involved in aggregation.
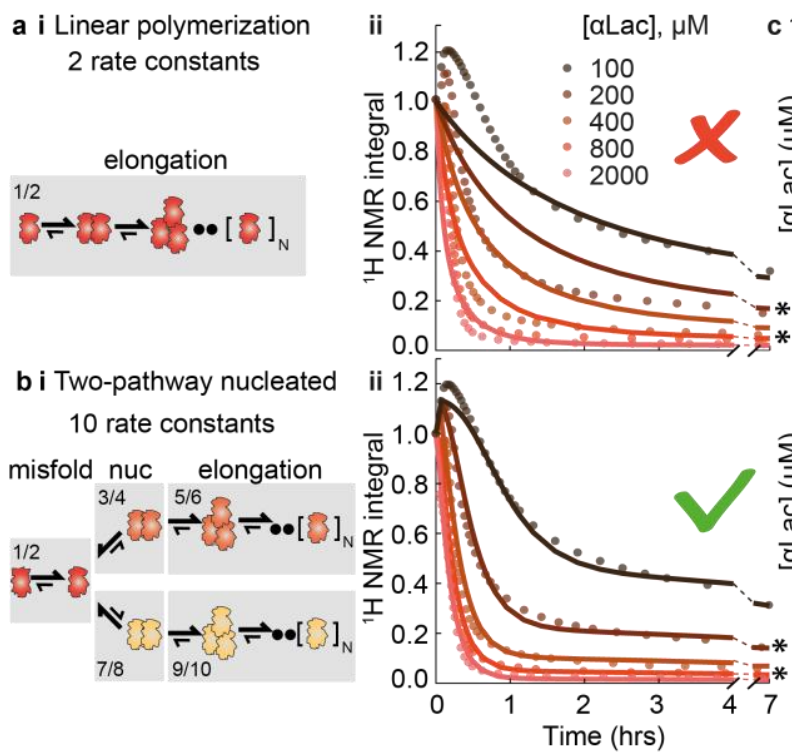

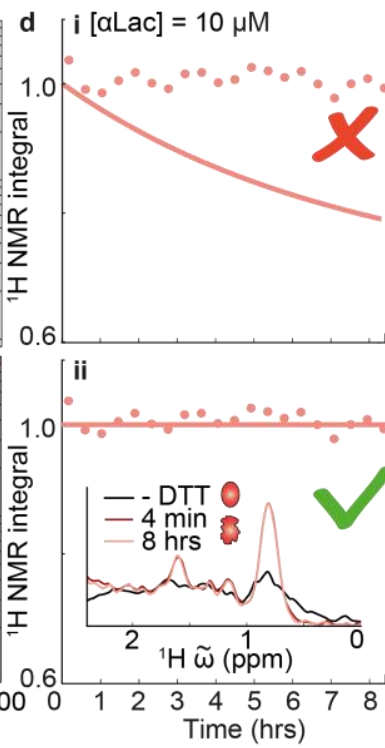

Figure 3 | Determining the mechanism of amyloid formation by aLac. a i The simplest aggregation model, linear polymerisation, parameterised by two rate constants. ii Aggregation timecourses at five $\alpha$ Lac concentrations (circles) with the fitted linear model (lines), revealing a poor fit to the data. b i The optimal aggregation model determined by our analysis (Table S2), parameterised by 10 rate constants. ii The fit of the optimal model to the data. Concentrations marked with an asterisk (200 and $800 \mu \mathrm{M})$ were excluded from the analysis and simulated only using the final rate constants for validation purposes. c An aggregation surface describing the loss of free monomer as a function of time and total protein concentration for (i) the linear polymerization model and (ii) the optimal model. The $80 \%$ free monomer isochor indicating the onset of aggregation is plotted (black line). The expected timecourse at $10 \mu \mathrm{M} \alpha \mathrm{Lac}$ is indicated as a line. $\mathbf{d}$ The experimentally measured timecourse at $10 \mu \mathrm{M} \alpha \mathrm{Lac}$ (circles) and the predictions for (i) the linear polymerization model and (ii) the optimal model. The inset in ii shows an overlay of representative NMR spectra for this dataset, illustrating the absence of signal loss over time in accordance with our optimal model. 


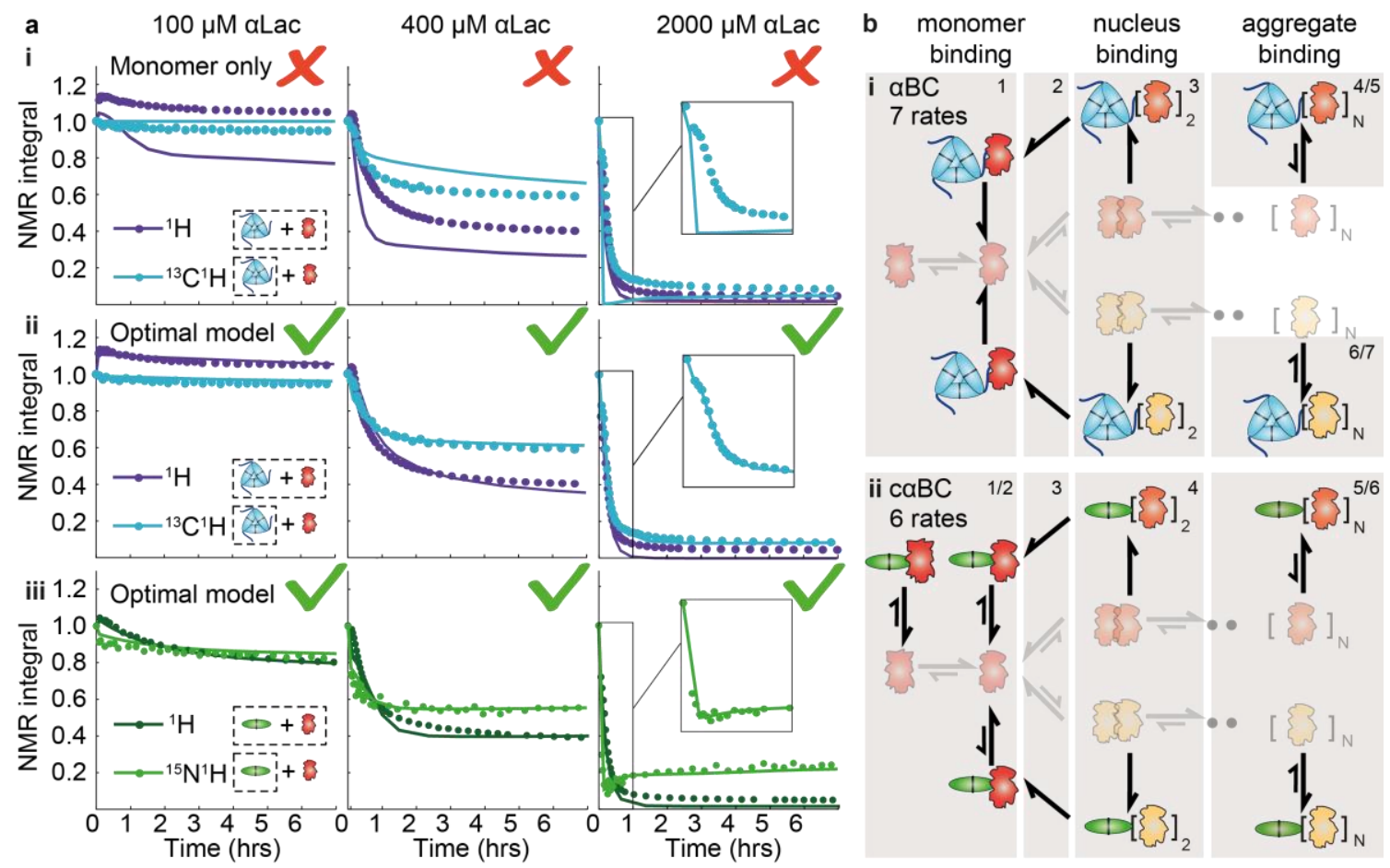

Figure 4 | Mechanism of $\alpha$ Lac amyloid inhibition by $\alpha B C$ and c $\alpha B C$. a NMR signal intensity timecourses for three concentrations of $\alpha \mathrm{Lac}$ in the absence and presence of either $\alpha \mathrm{BC}$ or c $\alpha \mathrm{BC}$ at $200 \mu \mathrm{M}$. For each $\alpha$ Lac concentration, isotope-unedited ${ }^{1} \mathrm{H}$ NMR signal and ${ }^{15} \mathrm{~N}$ - $(\mathrm{c} \alpha \mathrm{BC})$ or ${ }^{13} \mathrm{C}$ $(\alpha \mathrm{BC})$ edited chaperone-only ${ }^{1} \mathrm{H}$ signal is shown as circles. Corresponding lines show fits from (i) a model where the chaperone can bind only monomeric $\alpha \mathrm{Lac}$, (ii) and (iii) optimal models for $\alpha \mathrm{BC}$ and $c \alpha B C$ respectively. $\mathbf{b}$ The corresponding mechanisms for the optimal models (i $-\alpha B C, \mathbf{i i}-c \alpha B C)$.

\section{Chemical kinetics modelling reveals that aLac aggregates via two parallel nucleated pathways}

To elucidate the quantitative mechanisms of amyloid formation and chaperone action from our data, we chose to develop a chemical kinetics modelling strategy combined with stepwise regression. In our approach, specific aggregation mechanisms of varying complexity are hypothesised, and simulated (Table S2). Each proposed mechanism predicts how the concentration of species of every stoichiometry varies with time for a given set of rate constants, allowing us to simulate the NMR timecourse data. The rate constants for a model are then optimised numerically via a multi-step stimulated annealing protocol to determine best-fit values and the lowest possible $\chi^{2}$ to the global dataset that the model could achieve. A combination of forward (increasing complexity) and reverse (reducing complexity) steps were used to compare and construct models, where complexity refers to 
the number of fitting parameters (Fig. S4). To avoid the inherent risks of over-fitting to which stepwise regression is prone, F-tests were performed to determine whether any improvement in fit with an increased number of parameters could be explained by noise in our data (P-level < 0.05). In addition, to test the resulting mechanisms, a subset of data was deliberately excluded from model building and was subsequently simulated using the parameters obtained from the main set of data.

We first employed this approach to investigate the aggregation of $\alpha \mathrm{Lac}$ in the absence of chaperone. The simplest model for amyloid formation is linear polymerisation, which assumes that aggregation proceeds through consecutive monomer addition, and that all association and dissociation rates of oligomers are independent of stoichiometry ${ }^{44}$. This model, parameterised by two rate constants, provided a poor description of the data, and could be confidently excluded (Fig 3a, Table S2, model 1). We then built additional complexity into the model, including effects such as amyloid formation proceeding via an aggregation-prone monomer form, fragmentation of the aggregates, variations in the size of the aggregation nucleus, spherical rather than linear aggregate growth, and multiple aggregation pathways (Table S2). Overall, we tested 17 mechanisms of varying complexity until we identified a model that explained our data well, and where increasing the model complexity further did not result in a statistical improvement.

Achieving a satisfactory global fit was remarkably challenging (Fig. 3a, Table S2), with low complexity models unable to explain the data, revealing the high information content of the NMR data. A model of intermediate complexity, characterised by 10 rate constants was identified as our optimum in terms of lowered $\chi^{2}$ values versus the increase in the number of model parameters. This model includes formation of an on-pathway aggregation-prone monomeric species, and two parallel nucleated aggregation pathways (Table S2, Fig. S4, S6). Each of these aspects can be related directly to features in the raw data: without including an aggregation-prone monomer, we could not account for the increase in signal at early times; without nucleation we could not properly account for the concentration dependence of signal decay rate at early times; and without two pathways we could not account for the bi-phasic decay of signal at long times (Fig. 3b). The interrogation of the NMR 
aggregation curves using chemical kinetics therefore provides an in-depth, quantitative understanding of the $\alpha$ Lac aggregation mechanism, identifying and characterising key species (Fig. 3b).

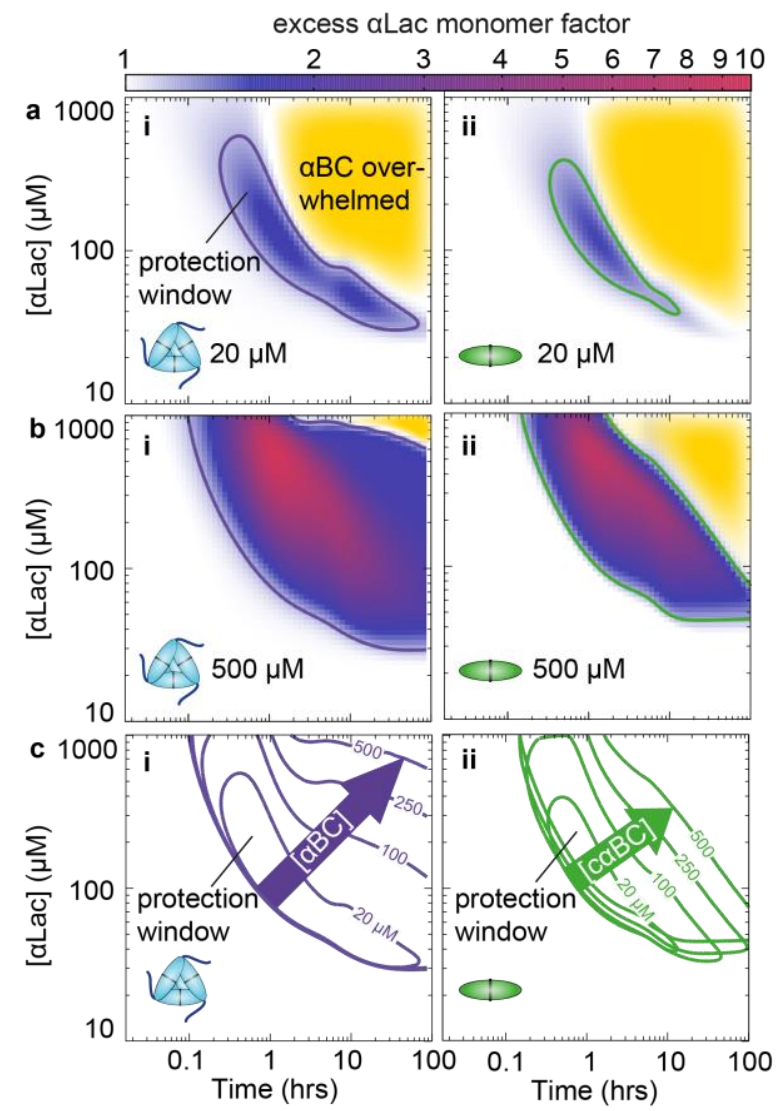

Figure 5 | Effect of $\alpha \mathrm{BC}$ (i) and $\mathrm{c} \alpha \mathrm{Bc}$ (ii) on aLac monomer. Protection is expressed as the fold excess $\alpha$ Lac monomer, versus total concentration of $\alpha \mathrm{Lac}$ and time for $20 \mu \mathrm{M}$ (a) and $500 \mu \mathrm{M}(\mathbf{b})$ chaperone. In each case, a window of monomer protection is revealed, and at longer times, the thermodynamically favoured aggregates dominate (yellow). Although their action is predominantly kinetic, both chaperones attenuate the point of onset of aggregation significantly (see also Fig. S9). Contour lines are drawn at the value of 1.2 (20\% increase in free $\alpha$ Lac monomer). $\mathbf{c}$ The $20 \%$ increase contour line shown as a function of chaperone concentration, illustrating the window of $\alpha$ Lac monomer protection by the chaperones.

\section{$\alpha B C$ inhibits $\alpha$ Lac aggregation by disassembling aggregation nuclei}

Having determined the mechanism of $\alpha$ Lac aggregation, we sought to investigate how $\alpha B C$ effects inhibition of this process by applying our chemical kinetics approach to the data in the presence of chaperone. We performed extensive model testing to find one that could globally explain our data for each chaperone (Fig. 4, Fig. S4, Tables S3-5), simulating both the chaperone and aggregate data 'channels'. The parameters for $\alpha$ Lac aggregation were fixed to those derived in the analysis of $\alpha$ Lac alone (Fig. 3, Fig. S6). We then introduced additional steps involving interactions between chaperone and the various aggregate states of $\alpha$ Lac. 40 different association models parameterised by 2 to 12 rate constants were tested to determine if they can fit to the data (Tables S3-5). As with $\alpha$ Lac, forward selection was performed, where a series of models of increasing complexity were tested. This process identified complex models that produced good fits to the data. These models were then subjected to 
reductive elimination, where individual binding processes were excluded, to obtain a minimally complex model that explained the data adequately.

We first tested the hypothesis that reversible binding of the chaperone to various species along the aggregation pathway effectively stalls the aggregation process. We tested chaperone binding to all species equally, or separately to misfolded $\alpha$ Lac monomers, nuclei or aggregates. These simple models that allowed binding of one type of species, or increasingly complex models that included all combinations of binding effects were in poor agreement with the data (Fig. 4a i, Table S3). This revealed that the NMR data could not be explained only by competitive binding of the chaperone to aggregating $\alpha$ Lac. To account for the persistence of $\alpha$ Lac monomer signal in our data, we hypothesised that the chaperones could additionally disrupt the aggregation nuclei after binding (Fig. 4b and Fig. S7), resulting in chaperone-bound monomers, which can then dissociate. Remarkably, we found that models including this effect were in excellent agreement with our data for both $\alpha B C$ and $\mathrm{c} \alpha \mathrm{BC}$, at all concentrations surveyed and throughout the reductive elimination process (Tables S3-5, Fig 4a and Fig. S5). The final models, selected through our statistical criteria, required 6 rate constants for $\mathrm{c} \alpha \mathrm{BC}$, and 7 for $\alpha \mathrm{BC}$ to explain the entire NMR dataset (Fig. 4b, Fig. S8). For both chaperone forms, it was necessary to include binding to both the nucleus and higher order aggregates, and a pathway for nucleus disassembly. A notable difference between the two forms of the chaperone was that $c \alpha B C$ bound to all types of species, whereas $\alpha \mathrm{BC}$ bound only the aggregating oligomers of $\alpha \mathrm{Lac}$ but not the monomer, revealing it to be more selective than the truncated form.

To validate the final models, over and above the data-partitioning strategy (Fig. S5), we used the fitted parameters from the best-fitting models to test consistency with the form of the 2D NMR spectra, where both chaperones were observed to be in slow chemical exchange (Fig. S3). The fastest predicted exchange rate from our model was determined to be $0.2 \mathrm{~s}^{-1}$ (Section S1.8), which is consistent with the observed slow exchange. In addition, a notable prediction of the nucleated aggregation model of $\alpha \mathrm{Lac}$ is that $<10 \mu \mathrm{M}$, no significant aggregation will occur on the timescale of days. We independently tested this prediction, and found that this was the case (Fig. 3c-d). Finally, EM images obtained 12 hours after the addition of DTT show two clearly distinguishable 
morphologies of aggregate: large filamentous structures, and smaller spherical aggregates (Fig. $2 \mathbf{e}$ and Fig. S1c). This is consistent with prediction of two parallel aggregation pathways for $\alpha$ Lac (Fig. S6 $)^{33,45}$.

\section{$\alpha B C$ kinetically stabilises aggregating aLac}

Our final models for $\alpha \mathrm{BC} / \alpha \mathrm{Lac}$ interaction allow us to simulate the proportion of free $\alpha \mathrm{Lac}$ monomer expected at any given time and protein concentration, and how this is modulated by interaction with the chaperones. We defined an 'excess monomer factor' as the ratio of free $\alpha$ Lac present in solution relative to that expected in the absence of chaperone (Fig. 5). Calculation of the excess monomer factor, over a broad range of $\alpha$ Lac concentrations and as a function of time, reveals a bounded region that we term a 'protection window' (Fig. 5a). At early times, there is little aggregation, and hence no chaperone activity. In the protection window, both chaperones significantly delay the onset of aggregation (Fig. S9), leading to accumulation of additional $\alpha$ Lac monomer and therefore rescue factors above 1 on the timescale of minutes to hours (Fig. 5). Most notably, at longer times, this protection is lost, as the thermodynamically driven aggregation process ultimately overwhelms the chaperone. This occurs earlier for higher $\alpha$ Lac concentrations (Fig. 5). Interestingly, the chaperone is most effective when only a small fraction of it is bound at a given time, and becomes overwhelmed when it is almost fully bound (Fig. S9b), consistent with a kinetic effect. The protection window of $\alpha \mathrm{BC}$ was found to be significantly larger than that of $\mathrm{c} \alpha \mathrm{BC}$ at the same concentration, indicating that its increased selectivity and more fine-tuned association and dissociation rates (Fig. S8) make it ultimately a more effective chaperone. In terms of mechanism, nucleus disassembly and aggregate binding were the key chaperone actions giving rise to the protection window (Fig. S10). 


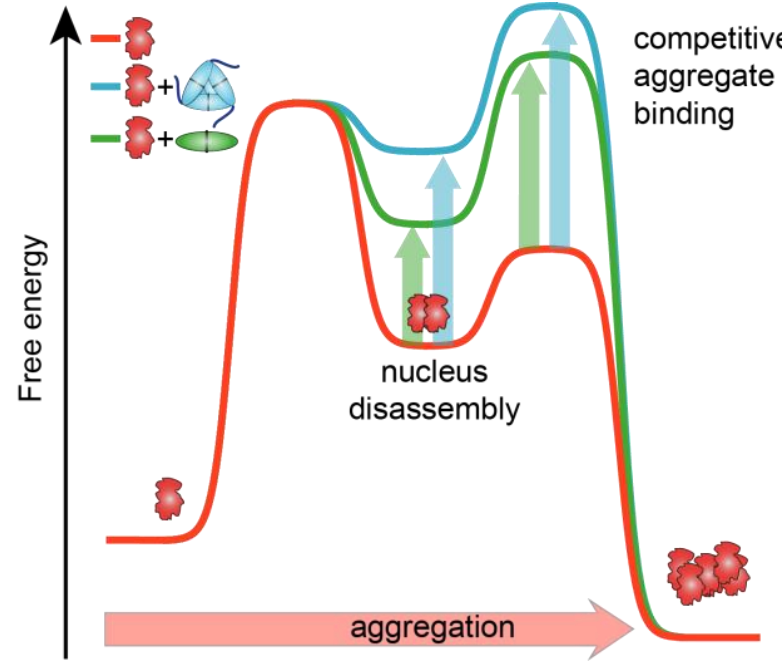

Figure 6 An illustrative summary of the aggregation inhibition mechanism of $\alpha \mathrm{BC}$ (blue) and caBC (green). As the concentrations of the relevant species vary over the course of the aggregation reaction, equilibrium free energy diagrams can only approximate the chemical changes. The kinetic barrier in $\alpha$ Lac aggregation is the formation of primary nuclei. Both chaperones effectively destabilise the primary nuclei by disassembling them, thus significantly slowing down the onset of aggregation. The chaperones also bind higher order aggregates to further slow their elongation. At long times, however, the thermodynamically favoured aggregation process eventually overwhelms the chaperones.

\section{Discussion}

The combined NMR and chemical kinetics methodology we have presented here provides a quantitative means for mechanistic characterisation of protein aggregation, and its inhibition. The overall form of the real-time data varies significantly with protein concentration, which lends the approach high discriminatory power necessary for mechanism determination. In addition, NMR spectra are sensitive to changes in monomer concentration at early times, during the lag phase of traditional assays based on light scattering, demonstrating that our approach is particularly suited to the analysis of early stages in protein aggregation, which is typically the period where toxic earlyaggregates are expected to form ${ }^{5}$. We capitalised on these capabilities to determine the mechanism by which $\alpha$ Lac forms amyloid, and how this is attenuated by both oligomeric $\alpha \mathrm{BC}$ and $\mathrm{c} \alpha \mathrm{BC}$ (Fig. 6). We tested a total of 57 models, requiring $>1500$ hours of CPU time, in order to find optimal fits to the data. These final models should be considered minimally complex, in the sense that we can unambiguously reject simpler models that lead to poor descriptions of the data, and on the other hand our data do not statistically justify the inclusion of further complexity. The final models therefore represent the most appropriate mechanisms, and crucially provide quantitative chemical explanations (in terms of rate constants) for the key features observed in the raw data.

Our analysis provides a mechanism for $\alpha$ Lac aggregation, where initial unfolding of the molten globule $^{16}$ to an unstable aggregation prone monomer is followed by formation of unstable aggregation 
nuclei with ca. 0.2-1.2 mM dissociation constants and association rate constants on the order of 200$700 \mathrm{M}^{-1} \mathrm{~s}^{-1}$ (Fig. S6). A consequence of this instability is that aggregation at low concentrations is very slow, but, once formed, the nuclei grow by elongation into aggregates with two distinct morphologies (Fig. S1), which has been observed previously ${ }^{33,45}$. The mechanism is reversible ${ }^{23}$ with a steady-state population of free, monomeric $\alpha$ Lac remaining even at long times. Non-equilibrium phase diagrams (Fig. 3c) illustrate how the proportion of monomer is expected to develop as a function of $\alpha$ Lac concentration and time.

We reveal that the chaperone has two major roles. The first, reported previously for $\alpha \mathrm{BC}$ for different substrates $^{20,21,32}$, is to bind larger aggregates and competitively stall their elongation. The second, more surprising observation is that $\alpha \mathrm{BC}$ binds and disassembles the unstable aggregation nuclei. Most remarkably, this is accomplished without the use of ATP, and so $\alpha \mathrm{BC}$ can be considered a passive disaggregase. Although examples of ATP-independent disaggregases are known ${ }^{46}$, and $\alpha \mathrm{BC}$ has been shown to promote dissociation of apolipoprotein C-II fibrils ${ }^{32}$ and $\beta 2$-microglobulin oligomers ${ }^{24}$, the specific destabilisation of nuclei is a previously unidentified function of sHSPs. The mechanism of nucleus disassembly provides an elegant means for curtailing amyloid formation, and, since lower molecular mass aggregates have been identified as more toxic than the higher molecular mass, mature aggregates ${ }^{5}$, this represents an efficient way for $\alpha \mathrm{BC}$ to exert its protective role. The ability of the chaperones to inhibit aggregation is limited, and at long times in our assays, the chaperones are overwhelmed as the thermodynamically favoured aggregation dominates. At a given concentration, this protection window represents a period of time during which the chaperone has kinetically stabilised the amyloidogenic protein. This is in line with the function of sHSPs acting as "paramedics", stabilising substrates for sufficient time such that ATP-dependent proteostatic mechanisms can be mobilised ${ }^{47}$.

In the main, sHSPs assemble into high molecular mass oligomers. Given that the truncated dimeric forms of the protein can also be potent chaperones, it is unclear why it is desirable for sHSPs to oligomerise. Here we demonstrate that the wild-type, evolutionarily optimised, oligomeric $\alpha \mathrm{BC}$ binds only the nuclei and higher-order aggregates whereas the truncated construct, $c \alpha \mathrm{BC}$, in addition binds 
free unaggregated $\alpha$ Lac monomers. In the cellular milieu, unproductive interactions with other cellular components would be undesirable. Overall, $\alpha \mathrm{BC}$ maintains a greater proportion of the client in non-aggregated, monomeric form over time compared to $\mathrm{c} \alpha \mathrm{BC}$, revealing it to be a more effective chaperone, and implicates involvement of the termini in chaperone activity.

In summary, our methodology has provided detailed insight into the mechanism by which $\alpha \mathrm{BC}$ prevents the amyloidogenesis of $\alpha$ Lac and revealed a new tactic used by chaperones, highlighting that cells have evolved a striking diversity of complementary mechanisms with which to target protein aggregation, both during ageing and times of acute stress. Our approach is suitable for the studies of a wide range of biomedical targets associated with aggregation and amyloid formation. 


\section{Materials and Methods}

\section{Protein preparation}

Full-length human $\alpha \mathrm{B}$-crystallin ( $\alpha \mathrm{BC}$, UniprotKB ID P02511) and the core domain construct (residues 68-153, c $\alpha$ BC) were prepared as described previously ${ }^{25,48}$ in either LB or M9 minimal media supplemented with ${ }^{15} \mathrm{NH}_{4} \mathrm{Cl}$ or $\left[\mathrm{U}_{-}{ }^{13} \mathrm{C}\right]$-glucose (Cambridge Isotope Laboratories). Bovine $\alpha$ lactalbumin was purchased from Sigma Aldrich (L6010).

\section{Aggregation reactions}

Aggregation reactions were performed at $37^{\circ} \mathrm{C}$ in $50 \mathrm{mM}$ sodium phosphate $\mathrm{pH} 7.0,100 \mathrm{mM} \mathrm{NaCl}$ and $2 \mathrm{mM}$ EDTA with $5 \% \mathrm{D}_{2} \mathrm{O}$. Aggregation was initiated by addition of $20 \mathrm{mM}$ DTT.

\section{NMR experiments}

NMR experiments were performed on a narrow-bore Varian 14.1 T NMR spectrometer with a 5-mm Z-axis gradient triple resonance room temperature probe for solution samples. The following parameters were typically used. ${ }^{1} \mathrm{H}$ watergate: 32 scans per fid, acquisition time $100 \mathrm{~ms}$, recycle delay 1 s. ${ }^{15} \mathrm{~N}^{1} \mathrm{H}$ 1D HSQCs: 72 scans per fid, acquisition time $67 \mathrm{~ms}$, recycle delay $1 \mathrm{~s}$, transmitter offset in ${ }^{15} \mathrm{~N}$ dimension $117 \mathrm{ppm} .{ }^{13} \mathrm{C}^{1} \mathrm{H}$ 1D constant-time HSQCs: 64 scans per fid, acquisition time $64 \mathrm{~ms}$, recycle delay $1 \mathrm{~s}$, transmitter offset in ${ }^{13} \mathrm{C}$ dimension $35 \mathrm{ppm}$. The duration of the experiments was 40 s for ${ }^{1} \mathrm{H}$ watergate, $81 \mathrm{~s}$ for ${ }^{15} \mathrm{~N}^{1} \mathrm{H}$ HSQC and $73 \mathrm{~s}$ for ${ }^{13} \mathrm{C}^{1} \mathrm{H}$ HSQC. Spectra were analyzed using NMRPipe $^{49}$ and Sparky ${ }^{50}$. Processing included digital water suppression where necessary, exponential apodization at $10 \mathrm{~Hz}$, phase correction and linear baseline correction. The following ranges were taken for integration (no buffer components present): ${ }^{1} \mathrm{H}$ spectra: $0.77-2.73 \mathrm{ppm},{ }^{15} \mathrm{~N}^{1} \mathrm{H}$ spectra: 6.5 $9.5 \mathrm{ppm},{ }^{13} \mathrm{C}^{1} \mathrm{H}$ spectra: $0.84-2.33 \mathrm{ppm}$.

Pulsed field gradient diffusion experiments were carried out over 11 gradient strengths with $72\left({ }^{1} \mathrm{H}\right.$ experiments), $240\left({ }^{15} \mathrm{~N}^{1} \mathrm{H}\right.$ experiments $)$ or $64\left({ }^{13} \mathrm{C}^{1} \mathrm{H}\right.$ experiments $)$ scans per transient and an acquisition time of $200 \mathrm{~ms}\left({ }^{1} \mathrm{H}\right)$, or $64 \mathrm{~ms}\left({ }^{15} \mathrm{~N}^{1} \mathrm{H}\right.$ or $\left.{ }^{13} \mathrm{C}^{1} \mathrm{H}\right)$ respectively. The maximum gradient strength was $60 \mathrm{G} \mathrm{cm}^{-1}$. The gradient duration was $2 \mathrm{~ms}\left({ }^{1} \mathrm{H}\right), 1.4 \mathrm{~ms}\left({ }^{15} \mathrm{~N}^{1} \mathrm{H}\right)$ or $1 \mathrm{~ms}\left({ }^{13} \mathrm{C}{ }^{1} \mathrm{H}\right)$ with a 
$200 \mathrm{~ms}$ delay between gradients. Each ppm point was analysed independently and the diffusion coefficient and its error were determined as the mean and standard deviation of a Gaussian distribution obtained from a histogram of measurements over the integrated ppm region.

\section{Simulations and model fitting}

Complete kinetic models were constructed to follow the change in concentration of individual oligomeric species. Up to 4,000 mers were individually considered with $10^{5}$ time steps. The models were parameterised by a number of rate constants (Section S1.5). The exact rate equations were integrated numerically and used to simulate the experimental data (Section S1.5). The free parameters were optimised using an algorithm that combines steepest descent (Levenberg-Marquardt) and simulated annealing protocols (Section S1.5). The $\chi^{2}$ values of the fits to the experimental NMR timecourses were each compared using the F-test ( $\mathrm{P}$ level $<0.05$, Section S1.7). The errors in the parameters were estimated by performing the fit for each of the five concentrations independently

\section{(Section S1.8).}

\section{Electron microscopy}

Transmission electron microscopy was performed on a Tecnai 12 (FEI) transmission electron microscope operated at $120 \mathrm{kV}$. Samples at $5 \mu \mathrm{M}$ in $5 \mu \mathrm{l}$ were loaded on glow-discharged carbon grids, washed with water and stained with $2 \%$ uranyl acetate for $10 \mathrm{~s}$. Images were acquired with a Gatan OneView CMOS camera.

\section{Acknowledgements}

Simulations and parameter optimisation was performed at the University of Oxford Advanced Research Computing (ARC) facility (http://dx.doi.org/10.5281/zenodo.22558). The EM work was performed at the Dunn School of Pathology Imaging Facility and we thank the facility manager Errin Johnson for guidance. The FTIR experiments were performed by Robert Jacobs at the Surface Analysis Facility, Department of Chemistry, University of Oxford. We thank Henrik Müller for help with the analysis of diffusion data and with performing the ThT assays. We thank the EPSRC grant 
$\mathrm{EP} / \mathrm{J} 01835 \mathrm{X} / 1$ and the BBSRC grant BB/J014346/1 for funding. OT holds a Lamb and Flag

Scholarship from St. John's College, University of Oxford. JLPB holds a Royal Society University

Research Fellowship, and AJB a David Phillips Fellowship from the Biotechnology and Biosciences

Research Council.

\section{References}

1. Hochberg, G. K. A. et al. The structured core domain of $\alpha \mathrm{B}$-crystallin can prevent amyloid fibrillation and associated toxicity. Proc. Natl. Acad. Sci. 111, E1562-70 (2014).

2. Baldwin, A. J. et al. The polydispersity of alphaB-crystallin is rationalized by an interconverting polyhedral architecture. Structure 19, 1855-1863 (2011).

3. WHO. Dementia: A Public Health Priority. (2012).

4. $\quad$ Eisenberg, D. \& Jucker, M. The amyloid state of proteins in human diseases. Cell 148, 1188 1203 (2012).

5. Chiti, F. \& Dobson, C. M. Protein Misfolding, Amyloid Formation, and Human Disease: A Summary of Progress Over the Last Decade. Annu. Rev. Biochem. 86, 27-68 (2017).

6. Powers, E. T., Morimoto, R. I., Dillin, A., Kelly, J. W. \& Balch, W. E. Biological and Chemical Approaches to Diseases of Proteostasis Deficiency. Annu. Rev. Biochem. 78, 959991 (2009).

7. Richter, K., Haslbeck, M. \& Buchner, J. The Heat Shock Response: Life on the Verge of Death. Mol. Cell 40, 253-266 (2010).

8. Klaips, C. L., Jayaraj, G. G. \& Hartl, F. U. Pathways of cellular proteostasis in aging and disease. J. Cell Biol. jcb.201709, (2017).

9. Carra, S. et al. The growing world of small heat shock proteins: from structure to functions. Cell Stress Chaperones (2017). doi:10.1007/s12192-017-0787-8

10. Ecroyd, H. \& Carver, J. A. Crystallin proteins and amyloid fibrils. Cell. Mol. Life Sci. 66, 6281 (2009).

11. Iwaki, T. et al. Accumulation of AlphaB-crystallin in central nervous system glia and neurons in pathologic conditions. Am. J. Pathol. 140, 345-356 (1992).

12. Renkawek, K., Voorter, C. E., Bosman, G. J., van Workum, F. P. \& de Jong, W. W. Expression of $\alpha \mathrm{B}$-crystallin in Alzheimer's disease. Acta Neuropathol. 87, 155-60 (1994).

13. Renkawek, K., Stege, G. J. J. \& Bosman, G. Dementia, gliosis and expression of the small heat shock proteins hsp27 and $\alpha$ B-crystallin in Parkinson's disease. Neuroreport 10, 2273-2276 (1999).

14. Renkawek, K., van Workum, F. P. \& de Jong, W. W. Immunohistochemical expression of $\alpha$ Bcrystallin and heat shock protein 27 in the brain in Down syndrome. Dev. Brain Dysfunct. 8, 35-39 (1995).

15. Bettelheim, F. A., Ansari, R., Cheng, Q. F. \& Zigler, J. S. The mode of chaperoning of dithiothreitol-denatured $\alpha$-lactalbumin by $\alpha$-crystallin. Biochem. Biophys. Res. Commun. 261, 
292-7 (1999).

16. Carver, J. A. et al. The interaction of the molecular chaperone $\alpha$-crystallin with unfolding $\alpha$ lactalbumin: A structural and kinetic spectroscopic study. J. Mol. Biol. 318, 815-827 (2002).

17. Smith, A. M., Jahn, T. R., Ashcroft, A. E. \& Radford, S. E. Direct Observation of Oligomeric Species formed in the Early Stages of Amyloid Fibril Formation using Electrospray Ionisation Mass Spectrometry. J. Mol. Biol. 364, 9-19 (2006).

18. Borzova, V. A. et al. Quantification of Anti-Aggregation Activity of Chaperones: A TestSystem Based on Dithiothreitol-Induced Aggregation of Bovine Serum Albumin. PLoS One 8, $1-18$ (2013).

19. Young, L. M. et al. Screening and classifying small-molecule inhibitors of amyloid formation using ion mobility spectrometry-mass spectrometry. Nat. Chem. 7, 73-81 (2015).

20. Waudby, C. A. et al. The interaction of $\alpha \mathrm{B}$-crystallin with mature $\alpha$-synuclein amyloid fibrils inhibits their elongation. Biophys. J. 98, 843-851 (2010).

21. Shammas, S. L. et al. Binding of the molecular chaperone $\alpha \mathrm{B}$-crystallin to $\mathrm{A} \beta$ amyloid fibrils inhibits fibril elongation. Biophys. J. 101, 1681-9 (2011).

22. Knowles, T. P. J. et al. Twisting transition between crystalline and fibrillar phases of aggregated peptides. Phys. Rev. Lett. 109, 1-5 (2012).

23. Baldwin, A. J. et al. Metastability of Native Proteins and the Phenomenon of Amyloid Formation. J. Am. Chem. Soc. 133, 14160-3 (2011).

24. Esposito, G. et al. Monitoring the interaction between $\beta 2$-microglobulin and the molecular chaperone $\alpha \mathrm{B}$-crystallin by NMR and mass spectrometry: $\alpha \mathrm{B}$-crystallin dissociates $\beta 2$ microglobulin oligomers. J. Biol. Chem. 288, 17844-17858 (2013).

25. Horwitz, J., Huang, Q.-L., Ding, L. \& Bova, M. P. Lens $\alpha$-crystallin: Chaperone-like properties. Methods Enzymol. Mol. Chaperones 290, 365-383 (1998).

26. Meisl, G. et al. Differences in nucleation behavior underlie the contrasting aggregation kinetics of the A $\beta 40$ and A $\beta 42$ peptides. Proc. Natl. Acad. Sci. U. S. A. 111, 9384-9 (2014).

27. Cohen, S. I. A. et al. A molecular chaperone breaks the catalytic cycle that generates toxic A $\beta$ oligomers. Nat. Struct. Mol. Biol. 22, 207-213 (2015).

28. Meisl, G. et al. Molecular mechanisms of protein aggregation from global fitting of kinetic models. Nat. Protoc. 11, 252-272 (2016).

29. Hochberg, G. K. A. \& Benesch, J. L. P. Dynamical structure of $\alpha$ B-crystallin. Prog. Biophys. Mol. Biol. 115, 11-20 (2014).

30. Haslbeck, M., Peschek, J., Buchner, J. \& Weinkauf, S. Structure and function of $\alpha$-crystallins: Traversing from in vitro to in vivo. Biochim. Biophys. Acta - Gen. Subj. 1860, 149-166 (2016).

31. Baldwin, A. J. et al. The polydispersity of $\alpha \mathrm{B}$-crystallin is rationalized by an interconverting polyhedral architecture. Structure 19, 1855-63 (2011).

32. Binger, K. J. et al. Avoiding the oligomeric state: $\alpha \mathrm{B}$-crystallin inhibits fragmentation and induces dissociation of apolipoprotein C-II amyloid fibrils. FASEB J. 27, 1214-1222 (2013).

33. Kulig, M. \& Ecroyd, H. The small heat-shock protein $\alpha B$-crystallin uses different mechanisms of chaperone action to prevent the amorphous versus fibrillar aggregation of $\alpha$-lactalbumin. 
Biochem. J. 448, 343-52 (2012).

34. Wider, G. \& Dreier, L. Measuring protein concentrations by NMR spectroscopy. J. Am. Chem. Soc. 128, 2571-2576 (2006).

35. Wijesinha-Bettoni, R., Dobson, C. M. \& Redfield, C. Comparison of the structural and dynamical properties of holo and apo bovine $\alpha$-lactalbumin by NMR spectroscopy. J. Mol. Biol. 307, 885-898 (2001).

36. Kuwajima, K., Ikeguchi, M., Sugawara, T., Hiraoka, Y. \& Sugai, S. Kinetics of Disulfide Bond Reduction in a-Lactalbumin by Dithiothreitol and Molecular Basis of Superreactivity of the Cys6-Cys 120 Disulfide Bond. Biochemistry 29, 8240-8249 (1990).

37. Carver, J. A. et al. The interaction of the molecular chaperone $\alpha$-crystallin with unfolding $\alpha-$ lactalbumin: A structural and kinetic spectroscopic study. J. Mol. Biol. 318, 815-827 (2002).

38. Rösner, H. I. \& Redfield, C. The Human $\alpha$-Lactalbumin Molten Globule: Comparison of Structural Preferences at pH 2 and pH 7. J. Mol. Biol. 394, 351-62 (2009).

39. Wilkins, D. K. et al. Hydrodynamic radii of native and denatured proteins measured by pulse field gradient NMR techniques. Biochemistry 38, 16424-16431 (1999).

40. Andrew Baldwin, C. J. et al. Harnessing NMR relaxation interference effects to characterise supramolecular assemblies. Chem. Commun. Chem. Commun 52, 7411-7518 (2016).

41. Carver, J. A., Aquilina, J. A., Truscott, R. J. W. \& Ralston, G. B. Identification by 1H NMR spectroscopy of flexible C-terminal extensions in bovine lens $\alpha$-crystallin. October 3, 143-145 (1992).

42. Baldwin, A. J. et al. Probing dynamic conformations of the high molecular weight $\alpha \mathrm{B}-$ crystallin heat shock protein ensemble by NMR spectroscopy. J. Am. Chem. Soc. (2012).

43. Alderson, T. R., Benesch, J. L. P. \& Baldwin, A. J. Proline isomerization in the C-terminal region of HSP27. Cell Stress Chaperones 22, 639-651 (2017).

44. Oosawa, F. \& Kasai, M. A theory of linear and helical aggregations of macromolecules. $J$. Mol. Biol. 4, 10-21 (1962).

45. Goers, J., Permyakov, S. E., Permyakov, E. A., Uversky, V. N. \& Fink, A. L. Conformational prerequisites for $\alpha$-lactalbumin fibrillation. Biochemistry 41, 12546-51 (2002).

46. Jaru-Ampornpan, P. et al. Mechanism of an ATP-independent Protein Disaggregase: II. Distinct molecular interactions drive multiple steps during aggregate disassembly. J. Biol. Chem. 288, 13431-13445 (2013).

47. Hilton, G. R., Lioe, H., Stengel, F., Baldwin, A. J. \& Benesch, J. L. P. Small heat-shock proteins: paramedics of the cell. Top. Curr. Chem. 328, 69-98 (2013).

48. Laganowsky, A. et al. Crystal structures of truncated $\alpha \mathrm{A}$ and $\alpha \mathrm{B}$ crystallins reveal structural mechanisms of polydispersity important for eye lens function. Protein Sci. 19, 1031-43 (2010).

49. Delaglio, F. et al. NMRPipe - a Multidimensional Spectral Processing System Based on Unix Pipes. J. Biomol. NMR 6, 277-293 (1995).

50. Lee, W., Tonelli, M. \& Markley, J. L. NMRFAM-SPARKY: Enhanced software for biomolecular NMR spectroscopy. Bioinformatics 31, 1325-1327 (2015). 